International Journal of Pure and Applied Mathematics

Volume 116 No. $1 \quad 2017,269-284$

ISSN: 1311-8080 (printed version); ISSN: 1314-3395 (on-line version)

url: http://www.ijpam.eu

doi: 10.12732/ijpam.v116i1.26

\title{
NUMERICAL SOLUTION OF UNSTEADY FLOW BETWEEN PARALLEL PLATES USING HAAR WAVELET-QUASILINEARIZATION METHOD
}

\author{
Harinakshi Karkera ${ }^{1}$, Nagaraj N. Katagi ${ }^{2} \S$ \\ ${ }^{1,2}$ Department of Mathematics \\ Manipal Institute of Technology \\ Manipal University \\ Manipal, 576 104, INDIA
}

\begin{abstract}
The present study is concerned with the analysis of unsteady flow of viscous incompressible fluid between parallel rectangular and circular plates. The Navier-Stokes equations modelling the flow are transformed into a fourth order non-linear ordinary differential equation using a similarity transformation. The resulting equation is solved by a new efficient numerical scheme based on Haar wavelet-quasilinearization method. The solution representing pressure gradient is obtained for large values of Reynolds number. A comparison with numerical results generated by MATLAB routine bvp4c demonstrates that the proposed method is more efficient and promising technique for solving highly nonlinear two-point boundary value problems. The absolute error and rate of convergence of numerical results are computed to test the applicability and accuracy of the method.
\end{abstract}

AMS Subject Classification: $34 \mathrm{~A} 34,34 \mathrm{~B} 15,65 \mathrm{~T} 60$

Key Words: Haar wavelets, quasilinearization, operational matrix, squeezing flow

\section{Introduction}

Unsteady flow in channels, pipes and fluid flow near an oscillating plate have been intensively studied over the past few years due to its practical importance in understanding several engineering and physiological flow problems. For in-

Received: January 31, 2017

Revised: $\quad$ August 29, 2017

Published: September 14, 2017

$\S_{\text {Correspondence author }}$ (c) 2017 Academic Publications, Ltd. url: www.acadpubl.eu 
stance, such flow of significance in lubrication is the squeezing flow between two parallel plates $[1,2,3]$. The problem of unsteady squeezing of viscous fluid between two plates occurs in unsteady loading. In the light of this application, many researchers have explored the effect of inertia on the bearing characteristics [4]. Chandrashekharan and Ramanaiah [5] investigated unsteady flow between two parallel rectangular and circular plates by considering all inertia terms and combined effect of inertia force is studied. Such problems admits similarity solution there by reducing unsteady Navier-Stokes equations to nonlinear ordinary differential equation. The non-linear nature of the modelled equation in most cases precludes its exact solution. Therefore different numerical and semi-numerical methods have been applied for providing approximate solutions such as Finite difference method (FDM), Finite element method (FEM), Perturbation method [6], Adomian decomposition method (ADM) [7], Homotopy analysis method (HAM) [8] etc. The classical discretization methods are powerful tools for solving differential equation but method fails to give the solution when singularity exists. Similarly, semi numerical methods, like perturbation, homotopy analysis methods requires perturbation parameter or the solution sought out to confirm the so-called rule of solution expression and the rule of coefficient ergodicity. That is where wavelets play an important role in obtaining the approximate solutions of such problems.

Several researchers $[9,10,11,12,13]$ have contributed to the present growth of mathematical analysis of different types of wavelets. In particular, Chen and Hasio [14] proposed the Haar wavelet which is mathematically the simplest among all the wavelet families. Following this pioneering work, Lepik $[15,16,17,18]$ introduced the numerical solution of differential equation using Haar Wavelet. A comprehensive survey on Haar wavelet methods are given in a paper by Hariharan and Kannan[19]. In a recent study, Kaur et al.[20] proposed a modified Haar wavelet method by introducing the quasilinearization approach to overcome the difficulties in solving the non-linear boundary value problems. Later, Saeed and Rehman [21] successfully used the Haar wavelet quasilinearization approach for the solution of non-linear fractional differential equations.

In the present study, we consider the problem of the unsteady flow of a viscous incompressible fluid between two parallel infinitely long rectangular plates and two parallel circular plates [5]. Such flow situations are frequently come across in lubrication and known as squeeze films. The objective of the paper is to discuss about flow structure for large Reynolds number $\alpha$. In this paper, we employ an efficient numerical scheme based on Haar wavelet in conjunction with quasilinearization process to tackle a fourth order non-linear differential 


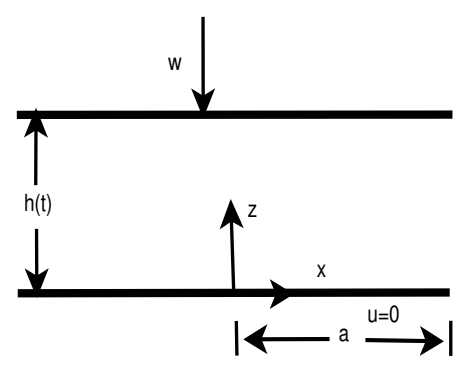

Figure 1: Schematic diagram of the problem (parallel rectangular plates or circular plates).

equation that governs the above problem. In the earlier study [22], this problem was analysed by perturbation method which is restricting the solution for moderately large values of $\alpha$. The proposed method does not pose any restriction on the parameter $\alpha$.

This paper is organized as follows: In Section 2, formulation of the problem is given. Section 3 contains the review of essential features of Haar wavelets, while in Section 4 basic ideas and principles of quasilinearization are discussed. Section 5 is devoted to implementation of the scheme to the given problem and efficiency of the method is discussed. The obtained results are discussed in Section 6 and we conclude our work in Section 7.

\section{Mathematical Formulation of the Problem}

\subsection{Two-Dimensional Flow}

Consider the viscous incompressible fluid between two parallel infinitely long rectangular plates of width $2 a$ separated by a small distance $h(t)$ as shown in Figure 1. It is assumed that the upper plate is moving downwards and the lower plate remains fixed [5]. Let $u$ and $w$ be the velocity components of the fluid in the $x$ and $z$ directions respectively. The two-dimensional governing equations of continuity and of motion are:

$$
\begin{aligned}
\frac{\partial u}{\partial x}+\frac{\partial w}{\partial z} & =0 \\
\frac{\partial u}{\partial t}+u \frac{\partial u}{\partial x}+w \frac{\partial u}{\partial z} & =-\frac{1}{\rho} \frac{\partial p}{\partial x}+\nu\left(\frac{\partial^{2} u}{\partial x^{2}}+\frac{\partial^{2} u}{\partial z^{2}}\right)
\end{aligned}
$$




$$
\frac{\partial w}{\partial t}+u \frac{\partial w}{\partial x}+w \frac{\partial w}{\partial z}=-\frac{1}{\rho} \frac{\partial p}{\partial z}+\nu\left(\frac{\partial^{2} w}{\partial x^{2}}+\frac{\partial^{2} w}{\partial z^{2}}\right)
$$

corresponding boundary conditions are

$$
\begin{aligned}
& u=w=0 \quad \text { at } z=0 \\
& u=0, \quad w=\dot{h} \text { at } z=h
\end{aligned}
$$

Introducing the similarity transformations [5]:

$$
\begin{aligned}
w & =-\psi(z, t)=-\frac{\nu}{h} F(\eta) \\
u & =x \frac{\partial \psi}{\partial z}=\frac{x \nu F^{\prime}}{h^{2}}
\end{aligned}
$$

where $\eta=\frac{z}{h(t)}$, we obtain

$$
\begin{aligned}
& \frac{\partial p}{\partial x}=-\frac{\rho \nu^{2} x}{h^{4}}\left(\alpha \eta F^{\prime \prime}+2 \alpha F^{\prime}+F^{\prime 2}-F F^{\prime \prime}-F^{\prime \prime \prime}\right) \\
& \frac{\partial p}{\partial \eta}=-\frac{\rho \nu^{2}}{h^{2}}\left(-\alpha \eta F^{\prime}-\alpha F+F F^{\prime}+F^{\prime \prime}\right)
\end{aligned}
$$

where $\alpha=-\frac{h \dot{h}}{\nu}$ is the local Reynolds number. Eliminating the pressure from (7) and (8), we obtain

$$
F^{\prime \prime \prime \prime}+F F^{\prime \prime \prime}-\alpha \eta F^{\prime \prime \prime}-F^{\prime} F^{\prime \prime}-3 \alpha F^{\prime \prime}=0
$$

The relevant boundary conditions are

$$
\begin{aligned}
& F=F^{\prime}=0 \quad \text { at } \eta=0 \\
& F=\alpha, \quad F^{\prime}=0 \text { at } \eta=1
\end{aligned}
$$

\subsection{Axisymmetric Flow}

Axisymmetric flow between two parallel circular plates of radius a separated by a small distance $h(t)$ is shown in Figure 1. The upper plate moves towards the lower plate which is fixed. In cylindrical coordinates $(x, \theta, z)$, with velocity components $u$ and $w$ in the radial and axial directions respectively [5]. The axisymmetric flow equations are:

$$
\frac{\partial}{\partial x}(x u)+\frac{\partial}{\partial z}(x w)=0
$$




$$
\begin{aligned}
\frac{\partial u}{\partial t}+u \frac{\partial u}{\partial x}+w \frac{\partial u}{\partial z} & =-\frac{1}{\rho} \frac{\partial p}{\partial x}+\nu\left(\frac{\partial^{2} u}{\partial x^{2}}+\frac{1}{x} \frac{\partial u}{\partial x}+\frac{\partial^{2} u}{\partial z^{2}}-\frac{u}{x^{2}}\right) \\
\frac{\partial w}{\partial t}+u \frac{\partial w}{\partial x}+w \frac{\partial w}{\partial z} & =-\frac{1}{\rho} \frac{\partial p}{\partial z}+\nu\left(\frac{\partial^{2} w}{\partial x^{2}}+\frac{1}{x} \frac{\partial w}{\partial x}+\frac{\partial^{2} w}{\partial z^{2}}\right)
\end{aligned}
$$

with the following boundary conditions

$$
\begin{aligned}
& u=w=0 \quad \text { at } \quad z=0 \\
& u=0, \quad w=\dot{h} \quad \text { at } \quad z=h
\end{aligned}
$$

Using the similarity transformations [5]:

$$
\begin{aligned}
w & =-\frac{2 \nu}{h} F(\eta) \\
u & =\frac{x \nu F^{\prime}}{h^{2}}
\end{aligned}
$$

where $\eta=\frac{z}{h(t)}$, the above equations reduces to:

$$
F^{\prime \prime \prime \prime}+2 F F^{\prime \prime \prime}-\alpha \eta F^{\prime \prime \prime}-3 \alpha F^{\prime \prime}=0
$$

and the boundary conditions are

$$
\begin{aligned}
& F=F^{\prime}=0 \quad \text { at } \eta=0 \\
& F=\alpha / 2, \quad F^{\prime}=0 \text { at } \eta=1
\end{aligned}
$$

\section{Haar Wavelets}

Wavelets are generally constructed through multiresolution analysis (MRA). Mathematically, MRA deals with the decomposition of whole function space into individual subspaces $V_{j} \subset V_{j+1}$ so that the space $V_{j+1}$ consists of all rescaled functions in $V_{j}$. This means, a decomposition of each function into components of different scales so that an individual component of the original function $f$ occurs in each subspace [23]. These components can describe finer and finer versions of the original function $f$.

Definition: A multiresolution analysis(MRA) is a sequence $\left\{V_{j}\right\}_{j \in \mathbb{Z}}$ of closed subspaces of $L^{2}(\mathbb{R})$, such that the following properties are satisfied: 
(a) The sequence is nested, i.e, for all $j \in \mathbb{Z}, V_{j} \subset V_{j+1}$

(b) The spaces are related to each other by dyadic scaling, i.e, $f(x) \in V_{j}$ iff $f\left(\frac{x}{2^{j}}\right) \in V_{0}\left(\right.$ or $f(x) \in V_{0}$ iff $\left.f\left(2^{j} x\right) \in V_{j}\right)$

(c) The union $\bigcup_{j \in \mathbb{Z}} V_{j}$ is dense in $L_{2}(\mathbb{R})$, i.e, $\overline{\bigcup_{j \in \mathbb{Z}} V_{j}}=L_{2}(\mathbb{R})$

(d) The intersection of the spaces is reduced to the null function, i.e, $\bigcap_{j \in \mathbb{Z}} V_{j}=$ $\{0\}$

(e) There exists a function $\phi \in V_{0}$ such that the set $\left\{\phi_{0, k}=\phi(x-k)\right\}_{k \in \mathbb{Z}}$ is a Riesz basis for $V_{0}$.

The function $\phi$ is called the scaling function.

The Haar scaling function is defined as

$$
h_{1}(x)= \begin{cases}1, & x \in[0,1) \\ 0, & \text { elsewhere }\end{cases}
$$

which forms a square wave with unit magnitude. The Haar mother wavelet is obtained as the linear combination of the Haar scaling function:

$$
\begin{aligned}
h_{2}(x)= & h_{1}(2 x)-h_{1}(2 x-1) \\
= & \begin{cases}1, & 0 \leq x<\frac{1}{2} \\
-1, & \frac{1}{2} \leq x<1 \\
0, & \text { elsewhere }\end{cases}
\end{aligned}
$$

All the other subsequent members in Haar wavelet family are constructed from translation and dilation of $h_{2}(x)$ :

$$
\begin{aligned}
h_{i}(x) & =h_{2}\left(2^{j} x-k\right) \\
& = \begin{cases}1, & x \in\left[\xi_{1}(i), \xi_{2}(i)\right) \\
-1, & x \in\left[\xi_{2}(i), \xi_{3}(i)\right) \\
0, & \text { elsewhere }\end{cases}
\end{aligned}
$$

where

$$
\xi_{1}(i)=\frac{k}{m}, \quad \xi_{2}(i)=\frac{k+0.5}{m}, \quad \xi_{3}(i)=\frac{k+1}{m}
$$

and $j=0,1, \ldots, J$ stand for the dilation parameter. As $j$ increases, the support decreases. The translation parameter $k=0,1, \ldots, m-1$ indicates the location of the particular square wave $\left(m=2^{j}\right)$ and $i=m+k+1$ is the wavelet number. 
The minimum value of $i$ is 2 (i,e. $m=1, k=0$ ) and the maximum value of $i$ is $2 M$ where $M=2^{J}$.

The integrals of the Haar functions are

$$
\begin{aligned}
& p_{1, i}(x)=\int_{0}^{x} h_{i}(t) d t, \\
& = \begin{cases}x-\xi_{1}(i) & \text { for } x \in\left[\xi_{1}(i), \xi_{2}(i)\right), \\
\xi_{3}(i)-x & \text { for } x \in\left[\xi_{2}(i), \xi_{3}(i)\right), \\
0 & \text { elsewhere }\end{cases} \\
& p_{2, i}(x)=\int_{0}^{x} p_{1, i}(t) d t, \\
& = \begin{cases}\frac{1}{2}\left(x-\xi_{1}(i)\right)^{2} & \text { for } x \in\left[\xi_{1}(i), \xi_{2}(i)\right), \\
\frac{1}{4 m^{2}}-\frac{1}{2}\left(\xi_{3}(i)-x\right)^{2} & \text { for } x \in\left[\xi_{2}(i), \xi_{3}(i)\right), \\
\frac{1}{4 m^{2}} & \text { for } x \in\left[\xi_{3}(i), 1\right) \\
0 & \text { elsewhere }\end{cases}
\end{aligned}
$$

Similarly,

$$
p_{l+1, i}(x)=\int_{0}^{x} p_{l, i}(t) d t, \quad l=2,3, \ldots
$$

To apply the Haar wavelets for the numerical solutions one must put them into a discrete form using collocation method in which the number of collocation points are doubled at each iteration [18]. The collocation points are $x_{l}=$ $(l-0.5) \Delta x, \quad l=1,2, \ldots, 2 M, \Delta x=1 / 2 M$.

The results can be formulated into matrix form as $H(i, l)=h_{i}\left(x_{l}\right), P_{\alpha}(i, l)=$ $p_{\alpha, i}\left(x_{l}\right), \alpha=1,2, \ldots$ These $2 M \times 2 M$ Haar matrices are sparse matrices and thus implementation of algorithms will be quite easier.

As the Haar wavelets forms a basis for $L^{2}[0,1)$, any function $y(x)$ which is square integrable in the interval $[0,1)$ can be decomposed as

$$
y(x)=\sum_{i=1}^{\infty} a_{i} h_{i}(x),
$$

where $a_{i}$ are the Haar wavelet coefficients given as

$$
a_{i}=2^{j} \int_{0}^{1} y(x) h_{i}(x) d x .
$$


The series expansion of $y(x)$ can be terminated at finite terms if $y(x)$ is a piecewise constant or it may be approximated as a piecewise constant for each subinterval.

\section{Quasilinearization}

The concept of quasilinearization technique was introduced by Bellman and Kalaba [24] for obtaining numerical solutions of individual or system of nonlinear differential equations. In quasilinearization technique, the nonlinear differential equation is solved recursively by a series of linear differential equations. This method not only linearizes the nonlinear equation but also provides a sequence of functions that converges to the solution of the original nonlinear equation which is very essential in practice. To explain the quasilinearization process let us consider the nonlinear $r^{\text {th }}$ order differential equation in the form

$$
L^{(r)} y(x)=f\left(y(x), y^{(1)}(x), y^{(2)}(x), \ldots, y^{(r-1)}(x), x\right)
$$

with $r$ boundary conditions

$$
g_{k}\left(y(a), y^{(1)}(a), y^{(2)}(a), \ldots, y^{(r-1)}(a)\right)=0, \quad k=1, \ldots, l
$$

and

$$
g_{k}\left(y(b), y^{(1)}(b), y^{(2)}(b), \ldots, y^{(r-1)}(b)\right)=0, \quad k=l+1, \ldots, r .
$$

Here $f$ is nonlinear function of $y(x)$ and its $r-1$ derivatives are $y^{(j)}(x), j=$ $1,2, \ldots, r-1$ and $L^{(r)}$ is the linear $r^{\text {th }}$ order differential operator. The quasilinearization procedure determines the $(n+1)^{\text {th }}$ iterative approximation $y_{n+1}(x)$ to the solution of $r^{\text {th }}$ order nonlinear ordinary differential equation (22) as a solution of linear differential equation

$$
\begin{aligned}
& L^{(r)} y_{n+1}(x)=f\left(y_{n}(x), y_{n}^{(1)}(x), y_{n}^{(2)}(x), \ldots, y_{n}^{(r-1)}(x), x\right) \\
& \quad+\sum_{j=0}^{r-1}\left(y_{n+1}^{(j)}(x)-y_{n}^{(j)}(x)\right) f_{y^{(j)}}\left(y_{n}(x), y_{n}^{(1)}(x), \ldots, y_{n}^{(r-1)}(x), x\right)
\end{aligned}
$$

where $y_{n}^{0}(x)=y_{n}(x)$. The functions $f_{y^{(j)}}=\frac{\partial f}{\partial y^{(j)}}$ are functional derivatives of the functional

$$
f\left(y(x), y^{(1)}(x), y^{(2)}(x), \ldots, y^{(r-1)}(x), x\right)=0
$$


The general conditions for the convergence of the quasilinearization method for solving nonlinear ordinary differential equations derived in [25]. In this method, the zeroth approximation $y_{0}(x)$ can be obtained from physical or mathematical considerations of given problem.

\section{Method of Solution}

Equations (9) and (17) with the respective boundary conditions can be put in the general form as

$$
F^{\prime \prime \prime \prime}+A F F^{\prime \prime \prime}-\alpha \eta F^{\prime \prime \prime}-B F^{\prime} F^{\prime \prime}-3 \alpha F^{\prime \prime}=0
$$

subject to conditions

$$
\begin{aligned}
& F=F^{\prime}=0 \quad \text { at } \eta=0 \\
& F=C \alpha, \quad F^{\prime}=0 \text { at } \eta=1
\end{aligned}
$$

where $A=1, B=1, C=1$ corresponds to two-dimensional flow and $A=$ $2, B=0, C=\frac{1}{2}$ for axisymmetric flow.

On implementing quasilinearization method, (23) reduces to the linearized form as

$$
\begin{gathered}
F_{n+1}^{\prime \prime \prime \prime}+\left(A F_{n}-\alpha \eta\right) F_{n+1}^{\prime \prime \prime}-\left(B F_{n}^{\prime}+3 \alpha\right) F_{n+1}^{\prime \prime}-B F_{n}^{\prime \prime} F_{n+1}^{\prime}+A F_{n}^{\prime \prime \prime} F_{n+1} \\
=A F_{n} F_{n}^{\prime \prime \prime}-B F_{n}^{\prime} F_{n}^{\prime \prime}
\end{gathered}
$$

with corresponding boundary conditions

$$
\begin{aligned}
& F_{n+1}(0)=F_{n+1}^{\prime}(0)=0 \\
& F_{n+1}(1)=C \alpha, \quad F_{n+1}^{\prime}(1)=0
\end{aligned}
$$

We seek the solution of (25) by using the approach recommended by Chen and Hsiao [14]. That is, instead of solution of the differential equation its highest derivative is expanded into the Haar series.

$$
F_{n+1}^{\prime \prime \prime \prime}(\eta)=\sum_{i=1}^{2 M} a_{i} h_{i}(\eta)
$$

This approximation is integrated while the boundary conditions are incorporated by using integration constants. We derive the lower order derivatives as follows:

$$
F_{n+1}^{\prime \prime \prime}(\eta)=\sum_{i=1}^{2 M} a_{i} p_{1, i}(\eta)+F_{n+1}^{(3)}(0)
$$




$$
\begin{gathered}
F_{n+1}^{\prime \prime}(\eta)=\sum_{i=1}^{2 M} a_{i} p_{2, i}(\eta)+F_{n+1}^{(2)}(0)+\eta F_{n+1}^{(3)}(0) \\
F_{n+1}^{\prime}(\eta)=\sum_{i=1}^{2 M} a_{i} p_{3, i}(\eta)+\eta F_{n+1}^{(2)}(0)+\frac{\eta^{2}}{2} F_{n+1}^{(3)}(0) \\
F_{n+1}(\eta)=\sum_{i=1}^{2 M} a_{i} p_{4, i}(\eta)+\frac{\eta^{2}}{2} F_{n+1}^{(2)}(0)+\frac{\eta^{3}}{6} F_{n+1}^{(3)}(0)
\end{gathered}
$$

The unknown terms $F_{n+1}^{(2)}(0), F_{n+1}^{(3)}(0)$ in the above equations are evaluated and given by

$$
\begin{gathered}
F_{n+1}^{(2)}(0)=6 C \alpha+2 \sum_{i=1}^{2 M} a_{i} E_{2, i}-6 \sum_{i=1}^{2 M} a_{i} E_{3, i} \\
F_{n+1}^{(3)}(0)=-12 C \alpha-6 \sum_{i=1}^{2 M} a_{i} E_{2, i}+12 \sum_{i=1}^{2 M} a_{i} E_{3, i}
\end{gathered}
$$

where

$$
\begin{aligned}
& E_{2, i}=\int_{0}^{1} p_{2, i}(t) d t \\
& E_{3, i}=\int_{0}^{1} p_{3, i}(t) d t
\end{aligned}
$$

Substituting (27)-(33) in (25) and simplifying we obtain the following system of $2 M$ number of algebraic equations:

$$
\begin{aligned}
& \sum_{i=1}^{2 M} a_{i}\left[h_{i}(\eta)+\left(A F_{n}(\eta)-\alpha \eta\right)\left(p_{1, i}(\eta)-6 E_{2, i}+12 E_{3, i}\right)-\left(B F_{n}^{\prime}(\eta)+3 \alpha\right)\right. \\
&\left(p_{2, i}(\eta)+2 E_{2, i}-6 E_{3, i}-6 \eta E_{2, i}+12 \eta E_{3, i}\right)-B F_{n}^{\prime \prime}(\eta)\left(p_{3, i}(\eta)+2 \eta E_{2, i}-6 \eta E_{3, i}\right. \\
&\left.\left.-3 \eta^{2} E_{2, i}+6 \eta^{2} E_{3, i}\right)+A F_{n}^{\prime \prime \prime}(\eta)\left(p_{4, i}(\eta)+\eta^{2} E_{2, i}-3 \eta^{2} E_{3, i}-\eta^{3} E_{2, i}+2 \eta^{3} E_{3, i}\right)\right] \\
&= 18 C \alpha^{2}-48 C \alpha^{2} \eta+12 A C \alpha F_{n}(\eta)+6 B C \alpha F_{n}^{\prime}(\eta)-12 B C \alpha \eta F_{n}^{\prime}(\eta) \\
&+6 B C \alpha \eta F_{n}^{\prime \prime}(\eta)-6 B C \alpha \eta^{2} F_{n}^{\prime \prime}(\eta)-B F_{n}^{\prime}(\eta) F_{n}^{\prime \prime}(\eta)-3 A C \alpha \eta^{2} F_{n}^{\prime \prime \prime}(\eta) \\
&+2 A C \alpha \eta^{3} F_{n}^{\prime \prime \prime}(\eta)+A F_{n}(\eta) F_{n}^{\prime \prime \prime}(\eta)
\end{aligned}
$$

With the appropriate initial approximations, the above system is put into the matrix form by introducing the row vectors $\eta=[\eta(l)], a=\left[a_{i}\right]$ and the following $2 M \times 2 M$ matrices: $H=\left[h_{i}\left(\eta_{l}\right)\right], P_{1}=\left[p_{1, i}\left(\eta_{l}\right)\right], P_{2}=\left[p_{2, i}\left(\eta_{l}\right)\right], P_{3}=\left[p_{3, i}\left(\eta_{l}\right)\right], P_{4}=\left[p_{4, i}\left(\eta_{l}\right)\right]$. Solving the system (34) using MATLAB, we obtain the wavelet solution for different 
level of resolutions which are displayed in Tables 1 and 3 .

The applicability and efficiency of the Haar wavelet-quasilinearization method is demonstrated by estimating the error in the solution. The order of convergence [26] for $n$th order ordinary differential equation using Haar wavelets is $O\left[\left(\frac{1}{2^{J+1}}\right)^{2}\right]$, where $J$ is the resolution level. However, in the proposed study, the exact solution of the problem is not known and therefore we estimate the accuracy of the obtained approximate solution by using the following relation [16]

$$
\Delta(v)=\left|\frac{S(v+1)}{S(v)}-1\right|
$$

where $S(v)=\Delta \eta_{(v)} \sum_{i=1}^{2 M}\left|F_{(v)}\left(\eta_{i}\right)\right|$ and $\Delta \eta_{(v)}$ is the step size of the $v^{\text {th }}$ approximation. The convergence rate of the solution can be determined with the aid of the function

$$
\sigma(v)=\frac{\Delta(v-1)}{\Delta(v)}, \quad v=2,3, \ldots
$$

The absolute relative error and the rate of convergence for different values of $J$ are shown in Tables 2 and 4 . We also solved this problem by using MATLAB routine bvp4c, which is based on an adaptive Lobatto quadrature scheme [27], for comparing our results. The solution shown in Table 1 and 3 was obtained with the relative error tolerance on the residuals, RelTol $=10^{-6}$.

\section{Results and Discussion}

In this work, the novelty of Haar wavelet-quasilinearization method (HWQLM) in obtaining solution for fourth order non-linear ordinary differential equation representing unsteady flow between parallel plates is presented. The Haar series (31) gives the solution of the governing problem. The present analysis enables in obtaining the converging solution for the given governing problem. We have computed pressure gradient representing the series (28) for much larger value of $\alpha$. In this method we could able to find the solution for $\alpha \geq 300$ whereas in earlier findings [5, 22] they have obtained the solution only up to $\alpha=4$ and 60 respectively due to its limited validity and slow convergence of the series. The advantage of this method is that it has no restriction on the parameter $\alpha$. To check the accuracy of the HWQLM, comparison is made with the numerical solution obtained by MATLAB routine bvp4c (which fails to give converging solution for $\alpha>180$ ) and an excellent agreement was found. This reveals that the proposed method is more efficient and superior to the traditional numerical methods for the solution of nonlinear higher order boundary value problems. Moreover, quasilinearization process significantly reduces the number of iterations to obtain required accuracy of the solution (only four iterations). Comparison between the proposed Haar wavelet method (at different resolution levels $J$ ), bvp4c and previous findings $([5,22]$ ) for pressure gradient, $F^{\prime \prime \prime}(0)$, at selected $\alpha$ values are shown in Table 1 and 3 . We notice that pressure gradient increases considerably with increase in $\alpha$ in two-dimensional 
Table 1: Comparision of pressure gradient $\left(-F^{\prime \prime \prime}(0)\right)$ obtained using HWQLM, bvp4c with other methods (two-dimensional flow)

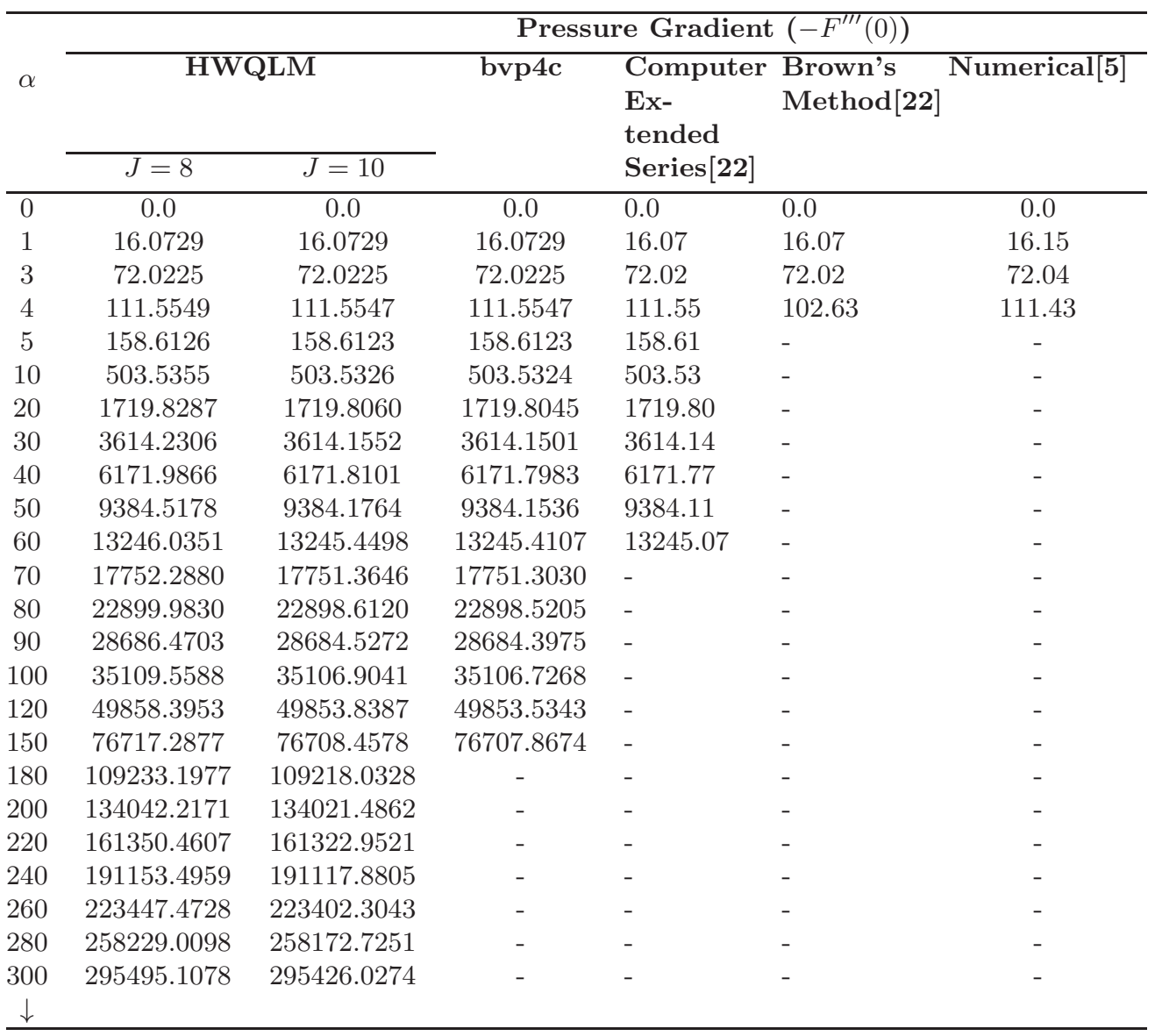

flow and axisymmetric flow. Also, for higher wavelet resolution level $J$, the method gives more accurate solutions. To validate our results obtained, we performed error analysis [16] and analysed that by doubling the number of collocation points the error roughly decreases four times. This theoretical estimation demonstrates the convergence of the numerical solution obtained by proposed method, which are shown in Table 2 and 4 for different values of $\alpha$. 
Table 2: Error estimates $\Delta$ and convergence rate $\sigma$ for two-dimensional flow case

\begin{tabular}{|c|c|c|c|c|c|c|c|c|}
\hline \multirow[b]{2}{*}{$J$} & \multicolumn{2}{|c|}{$\alpha=10$} & \multicolumn{2}{|c|}{$\alpha=20$} & \multicolumn{2}{|c|}{$\alpha=40$} & \multicolumn{2}{|c|}{$\alpha=60$} \\
\hline & $\Delta$ & $\sigma$ & $\Delta$ & $\sigma$ & $\Delta$ & $\sigma$ & $\Delta$ & $\bar{\sigma}$ \\
\hline 4 & $1.78 \mathrm{E}-16$ & - & $7.08 \mathrm{E}-13$ & - & $1.26 \mathrm{E}-10$ & - & $1.18 \mathrm{E}-09$ & - \\
\hline 5 & $1.78 \mathrm{E}-16$ & 1.00 & $1.54 \mathrm{E}-13$ & 4.59 & $2.13 \mathrm{E}-11$ & 5.90 & $1.28 \mathrm{E}-10$ & 9.25 \\
\hline 6 & 0 & - & $3.77 \mathrm{E}-14$ & 4.10 & $4.88 \mathrm{E}-12$ & 4.37 & $2.71 \mathrm{E}-11$ & 4.72 \\
\hline 7 & $1.07 \mathrm{E}-15$ & 0.00 & $9.41 \mathrm{E}-15$ & 4.00 & $1.20 \mathrm{E}-12$ & 4.08 & $6.51 \mathrm{E}-12$ & 4.16 \\
\hline 8 & $1.78 \mathrm{E}-15$ & 0.60 & $1.42 \mathrm{E}-15$ & 6.63 & $2.99 \mathrm{E}-13$ & 4.00 & $1.61 \mathrm{E}-12$ & 4.04 \\
\hline 9 & $2.13 \mathrm{E}-15$ & 0.83 & $3.55 \mathrm{E}-16$ & 4.00 & 7.19E-14 & 4.15 & $4.04 \mathrm{E}-13$ & 3.99 \\
\hline
\end{tabular}

\section{Conclusion}

The study confirms that the proposed HWQLM converges rapidly to the solution of original non-linear problem and can be used to solve many other non-linear differential equations arising in fluid mechanics and non-linear science in general. Another significant advantage of this method is that its implementation does not depend on small parameters unlike other traditional perturbation methods.

\section{References}

[1] P.S. Gupta, A.S. Gupta, Squeezing flow between parallel plates, Wear, 45, No. 2 (1977), 177-185, doi: 10.1016/0043-1648(77)90072-2.

[2] G. Ramanaiah, Inertia effects in hydromagnetic lubrication, Applied Scientific Research, 19, No. 1 (1968), 213-219, doi: 10.1007/BF00383923.

[3] P. Singh, V. Radhakrishnan, K.A. Narayan, Squeezing flow between parallel plates, Ingenieur-Archiv, 60, No. 4 (1990), 274-281, doi: 10.1007/BF00577864.

[4] J.D. Jackson, A study of squeezing flow, Applied Scientific Research, Section A, 11, No. 1 (1963), 148-152, doi: 10.1007/BF03184719.

[5] E. Chandrasekharan, G. Ramanaiah, Unsteady flow between rectangular plates and between circular plates, Wear, 91, No. 3 (1983), 307-318, doi: 10.1016/0043-1648(83)900753.

[6] M. Van dyke, Fluid dynamics off the real axis, Engineering Science, Fluid Dynamics, 1 (1990) 355-366.

[7] G. Adomian, Solving Frontier Problems in Physics: The decomposition method, Kluwer Academic Publishers, Netherlands (1994).

[8] S. Liao, Beyond Perturbation: Introduction to the Homotopy Analysis Method, Chapman \& Hall/CRC Press, Florida (2003).

[9] J. Biazar, H. Ebrahimi, Chebyshev wavelets approach for nonlinear systems of Volterra integral equations, Computers and Mathematics with Applications, 63, No. 3 (2012), 608-616, doi: 10.1016/j.camwa.2011.09.059. 
Table 3: Comparision of pressure gradient $\left(-F^{\prime \prime \prime}(0)\right)$ obtained using HWQLM, bvp4c with other methods (axisymmetric flow)

\begin{tabular}{|c|c|c|c|c|c|c|}
\hline \multirow{3}{*}{$\alpha$} & \multicolumn{6}{|c|}{ Pressure Gradient $\left(-F^{\prime \prime \prime}(0)\right)$} \\
\hline & \multicolumn{2}{|c|}{ HWQLM } & \multirow{2}{*}{ bvp4c } & \multirow{2}{*}{$\begin{array}{l}\text { Computer } \\
\text { Ex- } \\
\text { tended } \\
\text { Series }[22]\end{array}$} & \multirow{2}{*}{$\begin{array}{l}\text { Brown's } \\
\text { Method[22] }\end{array}$} & \multirow{2}{*}{ Numerical[5] } \\
\hline & $J=8$ & $J=10$ & & & & \\
\hline 0 & 0.0 & 0.0 & 0.0 & 0.0 & 0.0 & 0.0 \\
\hline 1 & 7.6599 & 7.6599 & 7.6599 & 7.659 & 7.659 & 7.72 \\
\hline 3 & 32.7540 & 32.7540 & 32.7539 & 32.75 & 32.75 & 32.95 \\
\hline 4 & 50.0826 & 50.0824 & 50.0824 & 50.08 & 50.08 & 50.69 \\
\hline 5 & 70.5403 & 70.5399 & 70.5399 & 70.53 & - & - \\
\hline 10 & 218.6203 & 218.6181 & 218.6179 & 218.61 & - & - \\
\hline 20 & 735.3927 & 735.3770 & 735.3760 & 735.37 & - & - \\
\hline 30 & 1536.4411 & 1536.3915 & 1536.3883 & 1536.38 & - & - \\
\hline 40 & 2615.3567 & 2615.2436 & 2615.2361 & 2615.23 & - & - \\
\hline 50 & 3968.3640 & 3968.1490 & 3968.1347 & 3968.12 & - & - \\
\hline 60 & 5592.9152 & 5592.5510 & 5592.5267 & 5592.47 & - & - \\
\hline 70 & 7487.1425 & 7486.5732 & 7486.5351 & - & - & - \\
\hline 80 & 9649.6004 & 9648.7613 & 9648.7052 & - & - & - \\
\hline 90 & 12079.1272 & 12077.9452 & 12077.8661 & - & - & - \\
\hline 100 & 14774.7625 & 14773.1559 & 14773.0483 & - & - & - \\
\hline 120 & 20961.2298 & 20958.4950 & 20958.3117 & - & - & - \\
\hline 150 & 32219.7247 & 32214.4748 & 32214.1216 & - & - & - \\
\hline 180 & 45841.5089 & 45832.5572 & 45831.9531 & & & \\
\hline 200 & 56230.8109 & 56218.6226 & - & - & - & - \\
\hline 220 & 67663.9076 & 67647.7918 & - & - & - & - \\
\hline 240 & 80138.8651 & 80118.0665 & - & - & - & - \\
\hline 260 & 93654.0054 & 93627.7045 & - & - & - & - \\
\hline 280 & 108207.8556 & 108175.1693 & - & - & - & - \\
\hline $\begin{array}{c}300 \\
\downarrow\end{array}$ & 123799.1107 & 123759.0928 & - & - & - & - \\
\hline
\end{tabular}

[10] I. Daubechies, Ten Lectures on Wavelets, Society for Industrial and Applied Mathematics, Pennsylvania (1992).

[11] L.A. Díaz, T.M. Martín, V. Vampa, Daubechies wavelet beam and plate finite elements, Finite Elements in Analysis and Design, 45, No. 3 (2009), 200-209, doi: 10.1016/j.finel.2008.09.006.

[12] A. Grossmann, J. Morlet, Decomposition of Hardy functions into square integrable wavelets of constant shape, SIAM Journal on Mathematical Analysis, 15, No. 4 (1984), 723-736, doi: 10.1137/0515056.

[13] H. Jafari, S.A. Yousefi, M.A. Firoozjaee, S. Momani, C.M. Khalique, Application of Legendre wavelets for solving fractional differential equations, Computers and Mathematics with Applications, 62, No. 3 (2011), 1038-1045, doi: 10.1016/j.camwa.2011.04.024. 
Table 4: Error estimates $\Delta$ and convergence rate $\sigma$ for axisymmetric flow case

\begin{tabular}{|c|c|c|c|c|c|c|c|c|}
\hline \multirow[b]{2}{*}{$J$} & \multicolumn{2}{|c|}{$\alpha=10$} & \multicolumn{2}{|c|}{$\alpha=20$} & \multicolumn{2}{|c|}{$\alpha=40$} & \multicolumn{2}{|c|}{$\alpha=60$} \\
\hline & $\Delta$ & $\sigma$ & $\Delta$ & $\sigma$ & $\Delta$ & $\bar{\sigma}$ & $\Delta$ & $\bar{\sigma}$ \\
\hline 4 & $5.68 \mathrm{E}-15$ & - & $1.22 \mathrm{E}-12$ & - & $7.93 \mathrm{E}-11$ & - & $1.17 \mathrm{E}-09$ & - \\
\hline 5 & $1.78 \mathrm{E}-15$ & 3.20 & $2.67 \mathrm{E}-13$ & 4.57 & $1.30 \mathrm{E}-11$ & 6.09 & $1.19 \mathrm{E}-10$ & 9.90 \\
\hline 6 & $3.55 \mathrm{E}-16$ & 5.00 & $6.43 \mathrm{E}-14$ & 4.15 & $2.97 \mathrm{E}-12$ & 4.38 & $2.51 \mathrm{E}-11$ & 4.73 \\
\hline 7 & $1.42 \mathrm{E}-15$ & 0.25 & $1.62 \mathrm{E}-14$ & 3.98 & $7.28 \mathrm{E}-13$ & 4.08 & $6.03 \mathrm{E}-12$ & 4.16 \\
\hline 8 & $1.07 \mathrm{E}-15$ & 1.33 & $4.44 \mathrm{E}-15$ & 3.64 & $1.79 \mathrm{E}-13$ & 4.06 & $1.49 \mathrm{E}-12$ & 4.04 \\
\hline 9 & $1.42 \mathrm{E}-15$ & 0.75 & $8.88 \mathrm{E}-16$ & 5.00 & $4.60 \mathrm{E}-14$ & 3.90 & $3.72 \mathrm{E}-13$ & 4.01 \\
\hline
\end{tabular}

[14] C.F. Chen, C.H. Hsiao, Haar wavelet method for solving lumped and distributedparameter systems, IEE Proceedings - Control Theory and Applications, 144, No. 1 (1997), 87-94, doi: 10.1049/ip-cta:19970702.

[15] Ü. Lepik, Numerical solution of differential equations using Haar wavelets, Mathematics and Computers in Simulation, 68, No. 2 (2005), 127-143, doi: 10.1016/j.matcom.2004.10.005.

[16] Ü. Lepik, Haar wavelet method for nonlinear integro-differential equations, Applied Mathematics and Computation, 176, No. 1 (2006), 324-333, doi: 10.1016/j.amc.2005.09.021.

[17] Ü. Lepik, Application of the Haar wavelet transform to solving integral and differential equations, Proceedings of the Estonian Academy of Sciences. Physics. Mathematics, 56, No. 1 (2007), 28-46.

[18] Ü. Lepik, Haar wavelet method for solving higher order differential equations, International Journal of Mathematics and Computation, 1, No. 8 (2008), 84-94.

[19] G. Hariharan, K. Kannan, An overview of Haar wavelet method for solving differential and integral equations, World Applied Sciences Journal, 23. No. 12 (2013), 01-14, doi: 10.5829/idosi.wasj.2013.23.12.423.

[20] H. Kaur, R.C. Mittal, V. Mishra, Haar wavelet quasilinearization approach for solving nonlinear boundary value problems, American Journal of Computational Mathematics, 1 (2011), 176-182, doi: 10.4236/ajcm.2011.13020.

[21] U. Saeed, M. Rehman, Haar wavelet-quasilinearization technique for fractional nonlinear differential equations, Applied Mathematics and Computation, 220 (2013), 630-648, doi: 10.1016/j.amc.2013.07.018.

[22] N.M. Bujurke, N.P. Pai, N.N. Katagi, V.B. Awati, Analytic continuation of series solution representing flow between plates, Indian Journal of Pure and Applied Mathematics, 35, No. 5 (2004), 587-598.

[23] L. Debnath, Wavelet Transforms and Their Applications, Birkhaüser, Boston (2002).

[24] R.E. Bellman, R.E. Kalaba, Quasilinearization and Nonlinear Boundary-Value Problems, American Elsevier Publishing Company, New York (1965).

[25] V.B. Mandelzweig, F. Tabakin, Quasilinearization approach to nonlinear problems in physics with application to nonlinear ODEs, Computer Physics Communications, 141, No. 2 (2001), 268-281, doi: 10.1016/S0010-4655(01)00415-5. 
[26] J. Majak, B.S. Shvartsman, M. Kirs, M. Pohlak, H. Herranen, Convergence theorem for the Haar wavelet based discretization method, Composite Structures, 126 (2015), 227-232, doi: 10.1016/j.compstruct.2015.02.050.

[27] L.F. Shampine, I. Gladwell, S. Thompson, Solving ODEs with MATLAB, Cambridge University Press, United Kingdom (2003). 